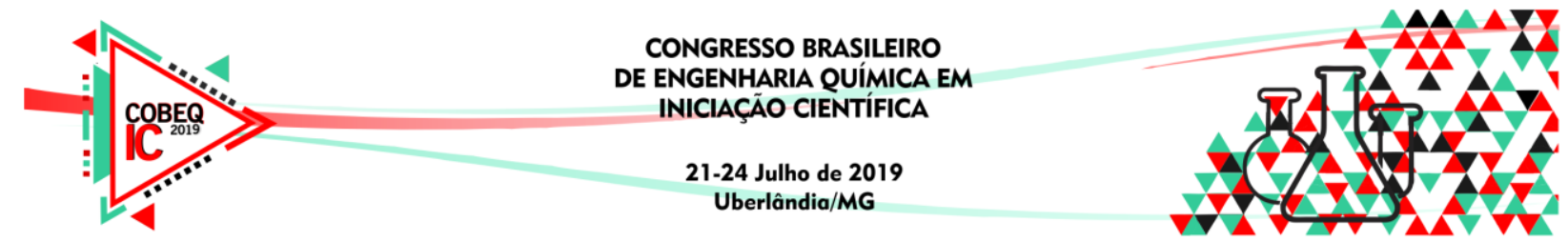

\title{
DADOS DE EQUILÍBRIO LÍQUIDO-LÍQUIDO DE SISTEMAS AQUOSOS CONTENDO LÍQUIDOS IÔNICOS PRÓTICOS E ACETONA
}

\author{
L. C. G. PETRIN ${ }^{1}$, F. P. PELAQUIM ${ }^{1}$, F. H. B. SOSA${ }^{1}$, R. M. DIAS ${ }^{1}$, M. C. $\operatorname{COSTA}^{1}$ \\ 1Universidade Estadual de Campinas, Faculdade de Engenharia Química \\ E-mail para contato: liviachagas98@gmail.com
}

\begin{abstract}
RESUMO - Nos últimos anos, os Líquidos Iônicos Próticos (LIPs) tem sido utilizados em diferentes áreas para uma série de aplicações, sendo considerados solventes promissores e de fácil síntese. Entretanto, após a sua aplicação, geralmente há a necessidade de se recuperar os LIPs e outros compostos que estão presentes no sistema. Para isso, em geral, são utilizadas etapas que envolvem equilíbrio de fases. Em vista disto, a determinação e descrição de dados de equilíbrio líquido-líquido (ELL) é necessária para o correto dimensionamento dos equipamentos, assim como para utilizar as condições que garantam a maior eficiência do processo. Neste contexto, neste trabalho foram determinados dados de ELL de dois sistemas aquosos formados por acetona e LIPs: formato de 2hidroxietanolamônio e acetato de 2-hidroxietanolamônio, na temperatura de 293.15 K e pressão atmosférica. As curvas binodais foram determinadas pelo método do ponto de névoa, e as linhas de amarração a partir do método gravimétrico. Além disso, os parâmetros do modelo NRTL foram ajustados utilizando os dados experimentais.
\end{abstract}

\section{INTRODUÇÃO}

Dados de ELL são necessários para o desenvolvimento de processos de separação (Fredj et al., 2015). Para determinação desses dados geralmente são usados solventes orgânicos ou sistemas aquosos bifásicos (SABs) (Huddelleston et al., 1998), com destaque para esse último por causar menos dano ao meio ambiente. Essa é uma das características que fez com que os sistemas aquosos fossem muito estudados nos últimos anos. Mais recentemente, a adição de novos solventes aos SABs - líquidos iônicos (LIs), por exemplo tem aberto novas possibilidades de aplicação desses sistemas para melhoria de processos de extração ou mesmo possibilitando a extração de compostos que antes não eram possíveis. Os LIs podem ser usados como adjuvantes o que ajudará o sistema a se dividir em duas fases ou ter uma região bifásica maior (Freire et al., 2012), além de aumentar a capacidade de separação de compostos de interesse em processos de partição (Souza et al., 2015).

Líquidos iônicos são considerados sais a temperatura ambiente, apresentando ponto de fusão abaixo de $100{ }^{\circ} \mathrm{C}$, e contêm ao menos um cátion orgânico e um ânion orgânico ou inorgânico (Losety et al., 2017). Além disso, são considerados solventes projetáveis, uma vez 


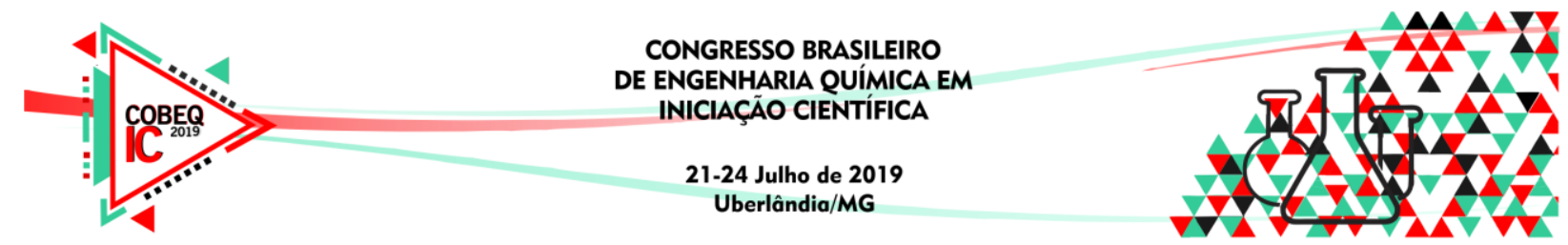

que podem ser formados a partir da combinação de cátions e ânions, que por sua vez alteram significativamente as suas propriedades físicas e químicas. São considerados não-inflamáveis, têm pressão de vapor baixa e também apresentam alta estabilidade química e térmica (Wasserscheid e Welton, 2008).

Os líquidos iônicos podem ser divididos em próticos (LIPs) ou apróticos (LIAs), e a chave para distinção deles é a permanência da carga positiva após a sua síntese. Para os LIPs, a carga positiva e as espécies neutras estão em equilíbrio, uma vez que eles são formados pela transferência de cargas positivas entre um ácido e uma base. O contrário é observado para os LIAs, nos quais a carga positiva é mantida no cátion e não há equilíbrio como aquele estabelecido para os LIPs. Este fato permite que os PILs apresentem vantagens no processo de preparação, uma vez que apenas uma reação ácido base é necessária, o que os tornam significativamente mais baratos que os LIAs, logo mais facilmente aplicados (Mirjafari et al., 2013).

De acordo com a literatura, estudos de ELL de sistemas aquosos envolvendo LIPs formados por etanolamina como base são escassos (Plácido et al., 2018). Em vista disto, o presente trabalho tem como objetivo determinar dados de ELL de sistemas aquosos envolvendo LIPs formados por monoetanolamina (base) e os ácidos fórmico e acético juntamente com acetona através da construção de curvas binodais e linhas de amarração. Além disso, os parâmetros do modelo NRTL (Renon e Prausnitz, 1968) foram ajustados utilizando os dados experimentais.

\section{MATERIAIS E MÉTODOS}

\subsection{Reagentes}

$\mathrm{Na}$ Tabela 1, são apresentados os reagentes utilizados neste estudo. Todos foram utilizados sem preparação prévia.

Tabela 1 - Reagentes utilizados nos experimentos

\begin{tabular}{|c|c|c|c|}
\hline Reagentes & Fornecedor & Pureza & Massa molar \\
\hline \hline Monoetanoalmina & Dinâmica & $99 \%$ & 61,08 \\
\hline Ácido Fórmico & Merck & $98 \%$ & 60,05 \\
\hline Ácido Acético & Anidrol & $99,7 \%$ & 60,05 \\
\hline Acetona & Anidrol & $99,5 \%$ & 58,08 \\
\hline
\end{tabular}

\subsection{Síntese dos LIPs}

Para a síntese dos LIPs, foram utilizados os ácidos fórmico e acético e a base monoetanolamina em uma reação de neutralização ácido-base na razão de equivalência molar de 1:1. A base foi inserida em um balão de vidro de 3 bocas equipado com um condensador de refluxo, um sensor PT-100 para monitorar a temperatura e um funil de decantação com gotejador. $\mathrm{O}$ ácido foi adicionado ao balão contendo a base de gota em gota. Durante todo o 


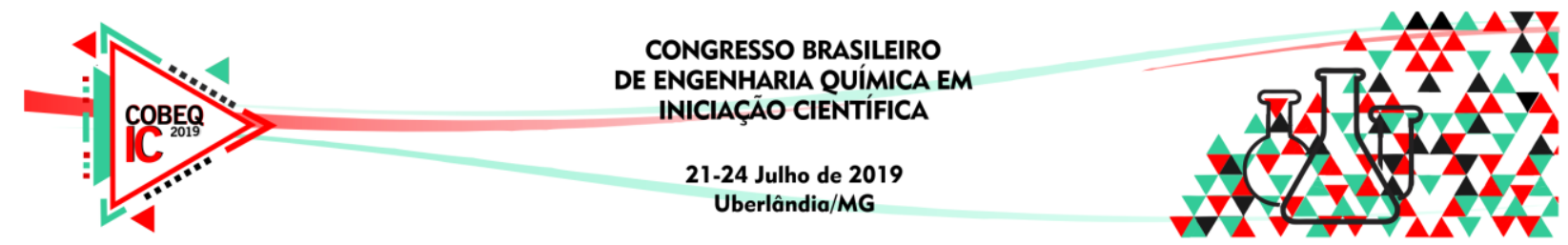

processo de gotejamento o balão foi mantido em contato com banho de gelo de forma a garantir a temperatura de $10^{\circ} \mathrm{C}$ e sob agitação com uso de um agitador mecânico.

Finalizado o gotejamento do ácido, a agitação foi mantida por 24 horas à temperatura ambiente. A etapa posterior consistiu da separação dos reagentes em excesso e da água absorvida pelo meio em um rotaevaporador conectado a uma bomba de vácuo (pressão absoluta de $70 \mathrm{~Pa}$ ). Esse processo também foi realizado com agitação vigorosa e aquecimento (entre $50^{\circ} \mathrm{C}$ e $60^{\circ} \mathrm{C}$ ) até que o teor de água no LI fosse menor que $1 \%$ medido através de um titulador Karl Fischer. Os LIPs sintetizados são: 2-HEAF [Formato de 2-hidroxietanolamônio (ácido fórmico + monoetanolamina)] e 2-HEAA [acetato de 2-hidroxietanolamônio (ácido acético + monoetanolamina)].

\subsection{Determinação das Curvas Binodais}

As curvas binodais foram determinadas para os sistemas 2-HEAF+água+acetona e 2HEAA+água+acetona a 293,15 K através do método de Ponto de Névoa (Kaul, 2000). As curvas binodais foram ajustadas através da equação 1 ( Merchuck et al. ,1998):

$$
Y=A \exp \left[\left(B X^{0,5}\right)+\left(C X^{3}\right)\right]
$$

Em que $Y$ é a fração mássica de LIPs e $X$ é a fração mássica de acetona, $A, B$ e $C$ são os parâmetros de Merchuck. Tais parâmetros foram obtidos a partir de ajuste utilizando a ferramenta Solver do software MS Excel 2013.

\subsection{Linhas de amarração (LAs)}

As linhas de amarração foram determinadas através do método gravimétrico proposto por Merchuck et al. (1998). Para tanto, cinco ou seis pontos de composição global situados na região bifásica foram escolhidos e preparados em tubos de centrífuga $(15 \mathrm{~mL})$ como previamente reportado por Sosa et al. (2017). Os tubos foram colocados em um banho termostático de temperatura constante $(293,15 \pm 0,05 \mathrm{~K})$ por 24 horas. A quantificação mássica das fases de topo e de fundo foi feita a partir de uma balança analítica com precisão de $0,0001 \mathrm{~g}$.

\subsection{Ajuste dos parâmetros de interação binária}

Os parâmetros de interação binária do modelo NRTL foram calculados utilizando o código Fortran TML-LLE (Stragevitch, 1997). Utilizou-se o método dos mínimos quadrados, que consiste na minimização da função objetivo:

$$
F O=\sum_{k}^{D} \sum_{j}^{M} \sum_{i}^{N-1}\left(w_{i j k}^{I \cdot \exp }-w_{i j k}^{I_{j} \cdot \operatorname{calc} c}\right)^{2}+\left(w_{i j k}^{I I \cdot \exp }-w_{i j k}^{I I \cdot c a l c}\right)^{2}
$$

Em que $D$ é o número de dados experimentais, $w$ é a fração mássica, $M$ e $N$ são o número de LAs e componentes, respectivamente, e $I$ e $I I$ se referem as duas fases líquidas em equilíbrio. 


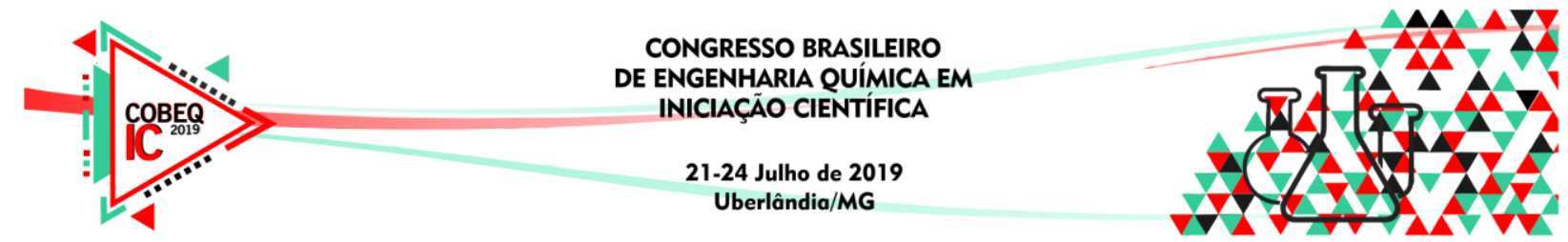

\section{RESULTADOS E DISCUSSÃO}

As curvas binodais, linhas de amarração e composição de ambas as fases para os sistemas 2-HEAF+água+acetona e 2-HEAA+água+acetona a 293,15 K e pressão atmosférica $(93,5 \mathrm{kPa})$ estão apresentados na Figura 1 e Tabela 2, e Figura 2 e Tabela 3, respectivamente. Os parâmetros $A, B$ e $C$ de Merchuck estão na Tabela 4.

As incertezas associadas às grandezas analisadas são: 0,05 K para temperatura, 0,0001 $\mathrm{g}$ para medidas mássicas e $0,5 \mathrm{kPa}$ para a pressão.

Figura 1 - (A) Curva binodal; (B) LA de (2-HEAF+água+acetona) a 293,15 K.

(A)

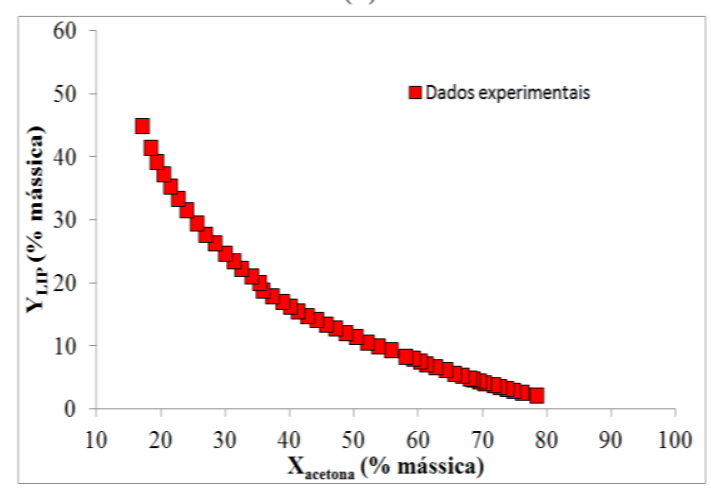

(B)

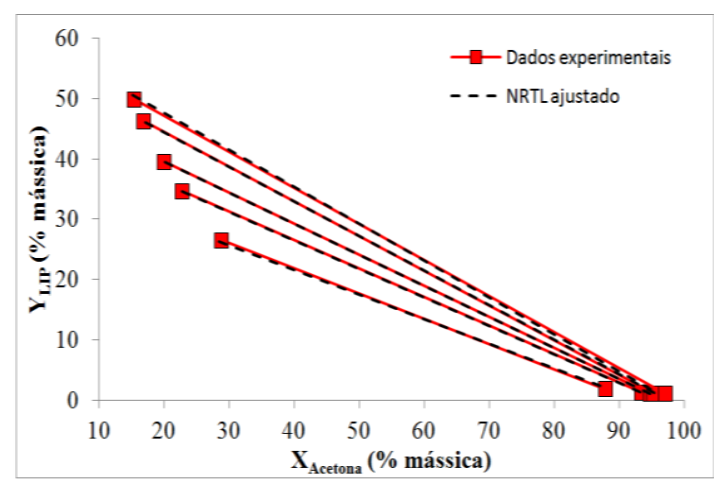

Figura 2 - (A) Curva binodal; (B) LA de (2-HEAA+água+acetona) a 293,15 K.

(A)

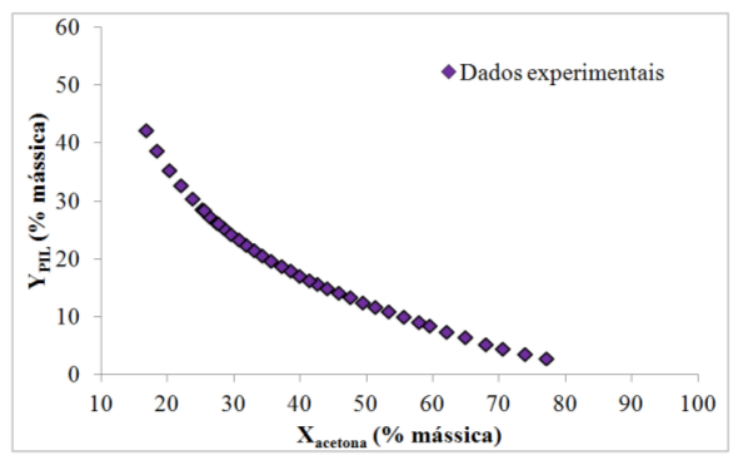

(B)

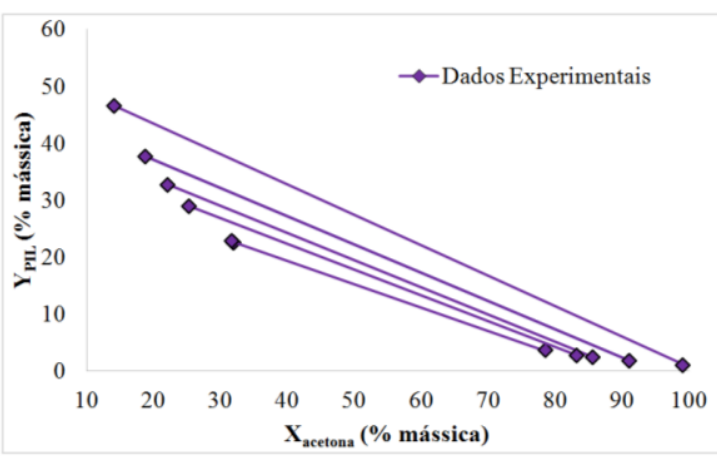

Tabela 2 - As composições globais e de cada fase para o sistema 2-HEAF+Acetona+Água a 293,15 K e 93,5 kPa.

\begin{tabular}{|c|c|c|c|c|c|c|c|c|c|}
\hline \multicolumn{4}{|c|}{ Composição global } & \multicolumn{3}{c|}{ Fase de topo } & \multicolumn{3}{c|}{ Fase de fundo } \\
\hline \hline LA & Acetona & 2-HEAF & Água & Acetona & 2-HEAF & Água & Acetona & 2-HEAF & Água \\
\hline 1 & 35,00 & 23,87 & 41,13 & 87,72 & 1,831 & 10,452 & 28,67 & 26,52 & 44,82 \\
\hline 2 & 36,96 & 27,86 & 35,05 & 93,31 & 1,312 & 5,380 & 22,60 & 34,70 & 42,70 \\
\hline 3 & 41,40 & 28,76 & 30,36 & 94,50 & 1,218 & 4,279 & 19,82 & 39,59 & 40,60 \\
\hline
\end{tabular}




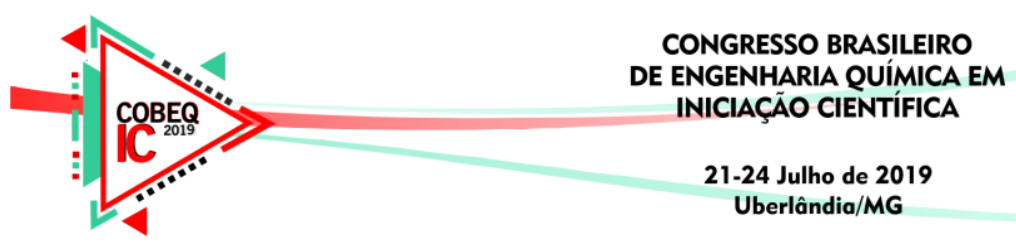

\begin{tabular}{|l|l|l|l|l|l|l|l|l|l|}
\hline 4 & 42,92 & 31,24 & 25,86 & 95,17 & 1,168 & 3,660 & 16,71 & 46,31 & 36,98 \\
\hline 5 & 44,97 & 32,11 & 22,82 & 97,06 & 1,035 & 1,907 & 15,31 & 49,90 & 34,79 \\
\hline
\end{tabular}

Tabela 3 - As composições globais e de cada fase para o sistema 2-HEAA+Acetona+Água a $293,15 \mathrm{~K} \mathrm{e} 93,5 \mathrm{kPa}$.

\begin{tabular}{|c|c|c|c|c|c|c|c|c|c|}
\hline & \multicolumn{3}{|c|}{ Composição global } & \multicolumn{3}{c|}{ Fase de topo } & \multicolumn{3}{c|}{ Fase de fundo } \\
\hline \hline LA & Acetona & 2-HEAA & Água & Acetona & 2-HEAA & Água & Acetona & 2-HEAA & Água \\
\hline 1 & 31,97 & 31,71 & 36,33 & 92,75 & 1,680 & 5,570 & 18,18 & 38,57 & 43,24 \\
\hline 2 & 29,87 & 28,95 & 41,00 & 85,53 & 2,506 & 11,97 & 22,10 & 32,74 & 45,17 \\
\hline 3 & 36,01 & 34,79 & 28,10 & 98,96 & 1,155 & 0,115 & 14,05 & 46,65 & 39,30 \\
\hline 4 & 55,92 & 19,15 & 24,46 & 90,93 & 1,680 & 7,392 & 18,69 & 37,73 & 43,58 \\
\hline 5 & 50,69 & 17,39 & 31,60 & 83,05 & 2,506 & 14,44 & 25,17 & 28,99 & 45,84 \\
\hline 6 & 48,06 & 15,97 & 35,55 & 78,33 & 1,155 & 20,52 & 31,56 & 22,82 & 45,62 \\
\hline
\end{tabular}

Tabela 4 - Parâmetros $A, B$ e $C$ da Equação de Merchuck et al. (equação 1) e R² de cada ajuste.

\begin{tabular}{|c|c|c|c|c|}
\hline Sistema & $A$ & $B$ & $C$ & $\mathrm{R}^{2}$ \\
\hline \hline 2-HEAF & 256,6919 & $-0,42264$ & $1,32 \mathrm{E}-06$ & 0,9985 \\
\hline 2-HEAA & 180,0721 & $-0,3592$ & $1,52 \mathrm{E}-06$ & 0,9982 \\
\hline
\end{tabular}

Os parâmetros de interação binária do modelo NRTL para o sistema 2HEAF+acetona+água estão apresentados na Tabela 5, calculado de acordo com a metodologia descrita na seção 2.5. O ajuste apresentou um desvio médio quadrático de 0,37\%.

Tabela 5 - Parâmetros $\mathrm{a}_{\mathrm{ij}}$ e $\alpha_{\mathrm{ij}}$ do modelo NRTL.

\begin{tabular}{|c|c|c|c|c|}
\hline $\mathrm{a}_{\mathrm{ij}}(\mathrm{J} / \mathrm{mol})$ & Acetona & 2-HEAF & Água & $\alpha_{\mathrm{ij}}$ \\
\hline \hline Acetona & 0 & 120,77 & 642,83 & 0,2 \\
\hline 2-HEAF & 541,41 & 0 & 273,87 & 0,45429 \\
\hline Água & 690,53 & $-1332,5$ & 0 & 0,22491 \\
\hline
\end{tabular}

Com base no exposto, as curvas binodais dos sistemas estudados foram determinadas pela metodologia do ponto de névoa. Além disso, foi possível determinar gravimetricamente as Linhas de Amarração, como descrito na Seção 2.4. Por fim, foi utilizado o modelo NRTL para ajustar os parâmetros de interação binária aos dados experimentais, apresentando um desvio médio quadrático de $0,37 \%$, o que demonstra a capacidade do modelo de descrever o comportamento do sistema aquoso formado por 2-HEAF+acetona. 


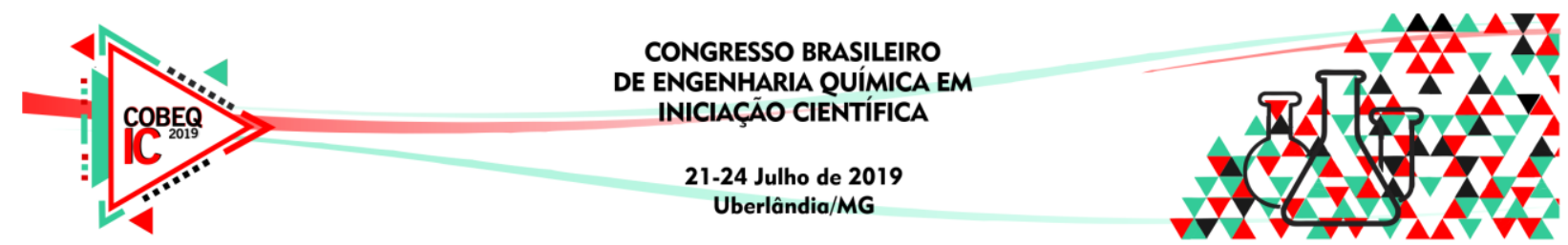

\section{REFERÊNCIAS}

FREDJ, S. B.; NABBS, J.; TIZAOUI, C.; MONSER, L. Removal of estrone (E1), 17 $\beta$ estradiol (E2), and 17 $\alpha$-ethinylestradiol (EE2) from wastewater by liquid-liquid extraction, Chem. Eng. J., v. 262, p. 417-426, 2015.

Freire, M. G., Cláudio, A. F. M., Araújo, J. M. M., Coutinho, J. A. P., Marrucho, I. M., Lopes, J. N. C., Rebelo, L. P. N. Aqueous biphasic systems: a boost brought about by using ionic liquids. Chem. Soc. Rev., 41, p. 4966-4995, 2012.

HUDDELESTON, J. G.;WILLAUER, H. D.; SWATLOSKI, R. P.; VISSER, A. E.;ROGER, R. D. Room temperature ionic liquid as novel media for "clean" liquid-liquid extraction, Chem. Commin., v. 16, p. 1765-1766, 1998.

KAUL R. H. Aqueous Two-Phase Systems, Methods and Protocols. New Jersey: Humana Press, 2000.

LOSETTY, V.; MATHESWARAN, P.; WILFRED, C.D. Synthesis, thermophysical properties and COSMO-RS study of DBU based protic ionic liquids, J. Chem. Thermodyn. 105, p. 151-158, 2017.

MERCHUK, J. C; ANDREWS, B. A.; ASENJO, J. A. Aqueous two-phase systems for protein separation. Studies on phase inversion. J. Chromatogr, v. 711, p. 285-293, 1998.

MIRJAFARI, A.; L. N. PHAM, L. N.; Mccabe, J. R.; MOBARREZ, N.; SALTER, E. A.; WIERZBICKI, A.; WEST, K. N.; SYKORA, R. E.; DAVIS, J. H. Building a bridge between aprotic and protic ionic liquids. RSC Adv., 3, p. 337-340, 2013.

NELDER, J. A.; MEAD, R. A Simplex method for function minimization. Comput. J., v. 7 p. $308-313,1965$.

PLÁCIDO, N. S. O.; CARLOS, A. L. S.; GALVÃO, J. U. S.; SOUZA, R. L.; SOARES, C. M. F.; MATTEDI, S.; FRICKS, A. T.; LIMA, Á. S. Protic ionic liquids as a constituent of biphasic systems based on acetonitrile: Phase diagram and alkaloid partitioning. Sep. and Pur. Technol., 200, p. 318-326, 2018.

RENON, H.; PRAUSNITZ, J. M. Local Compositions in Thermodynamic Excess Functions for Liquid Mixtures. AICHE Journal, v. 14 (1), p. 135-144, 1968.

SOSA, F. H. B.; DE ARAUJO SAMPAIO, D.; FARIAS, F. O.; BONASSOLI, A. B. G.; IGARASHI-MAFRA, L.; MAFRA, M. R. Measurement and correlation of phase equilibria in aqueous two-phase systems containing polyethyleneglycol $(2000,4000$, and 6 000) and sulfate salts (manganese sulfate and copper sulfate) at different temperatures $(298.15,318.15$, and 338.15 K). Fluid Ph. Equilibria, v.449, p. 68-75, 2017.

STRAGEVITCH, L.; D’ÁVILA, S. G. Application of a generalized maximum likelihood method in the reduction of multicomponent liquid-liquid equilibrium data. Braz. J. Chem. Eng., v. 14 (1), p., 1997.

WASSERSCHEID, P.; WELTON, T. Ionic liquids in synthesis, WILEY-VCH Verlag GmbH \& Co. KGaA, Weinheim, 2008. 